\title{
1 Nanoscopic resolution within a single imaging frame
}

2 Esley Torres García ${ }^{1,2}$, Raúl Pinto Cámara ${ }^{1,2}$, Alejandro Linares ${ }^{2}$, Damián Martínez ${ }^{2}$, Víctor Abonza ${ }^{2}$,

3 Eduardo Brito-Alarcón ${ }^{2}$, Carlos Calcines-Cruz ${ }^{3}$, Gustavo Valdés-Galindo ${ }^{4}$, David Torres ${ }^{2}$, Martina

4 Jabloñski ${ }^{5}$, Héctor H. Torres-Martínez ${ }^{6}$, José L. Martínez ${ }^{7}$, Haydee O. Hernández ${ }^{8}$, José P. Ocelotl-

5 Oviedo $^{2}$, Yasel Garcés ${ }^{2,7}$, Marco Barchi ${ }^{9}$, Joseph G. Dubrovsky ${ }^{6}$, Alberto Darszon ${ }^{7}$, Mariano G.

6 Buffone ${ }^{5}$, Roberto Rodríguez Morales ${ }^{10}$, Juan Manuel Rendon-Mancha ${ }^{1}$ Christopher D. Wood ${ }^{2}$,

7 Armando Hernández-García ${ }^{4}$, Diego Krapf ${ }^{11}$, Álvaro H. Crevenna ${ }^{12}$, and Adán Guerrero ${ }^{2, *}$.

8

$9 \quad{ }^{1}$ Centro de Investigación en Ciencias, Instituto de Investigación en Ciencias Básicas y Aplicadas,

10 Universidad Autónoma del Estado de Morelos, Cuernavaca, Morelos, Mexico.

$11{ }^{2}$ Laboratorio Nacional de Microscopía Avanzada, Instituto de Biotecnología, Universidad Nacional

12 Autónoma de México, Cuernavaca, Morelos, Mexico.

$13{ }^{3}$ Instituto de Investigaciones Biomédicas, Universidad Nacional Autónoma de México. Ciudad de

14 México, Mexico.

$15{ }^{4}$ Departamento de Química de Biomacromoléculas, Instituto de Química. Universidad Nacional

16 Autónoma de México. Ciudad de México, Mexico.

$17{ }^{5}$ Instituto de Biología y Medicina Experimental (IBYME-CONICET), Buenos Aires, Argentina.

$18{ }^{6}$ Departamento de Biología Molecular de Plantas, Instituto de Biotecnología, Universidad Nacional

19 Autónoma de México, Cuernavaca, Mexico.

$20{ }^{7}$ Departamento de Genética del Desarrollo y Fisiología Molecular, Instituto de Biotecnología,

21 Universidad Nacional Autónoma de México, Cuernavaca, Morelos, Mexico.

$22{ }^{8}$ Instituto de Investigaciones en Matemáticas Aplicadas y en Sistemas, Universidad Nacional Autónoma

23 de México, Ciudad de México, Mexico.

$24{ }^{9}$ Department of Biomedicine and Prevention, Faculty of Medicine, University of Rome Tor Vergata,

25 Rome, Italy.

$26{ }^{10}$ Instituto de Cibernética, Matemática y Física, Ciudad de la Habana, Cuba.

$27{ }^{11}$ Electrical and Computer Engineering and School of Biomedical Engineering, Colorado State

28 University, Fort Collins, Colorado 80523, U.S.A.

$29{ }^{12}$ European Molecular Biology Laboratory, Neurobiology and Epigenetics Unit, Monterotondo, Italy.

30

$31 *$ Correspondence contact: adan.guerrero@ibt.unam.mx 


\section{Abstract}

33 Mean-Shift Super Resolution (MSSR) is a principle based on the Mean Shift theory that

34 improves the spatial resolution in fluorescence images beyond the diffraction limit.

35 MSSR works on low- and high-density fluorophore images, is not limited by the

36 architecture of the detector (EM-CCD, sCMOS, or photomultiplier-based laser scanning

37 systems) and is applicable to single images as well as temporal series. The theoretical

38 limit of spatial resolution, based on optimized real-world imaging conditions and analysis of temporal image series, has been measured to be $40 \mathrm{~nm}$. Furthermore, MSSR has denoising capabilities that outperform other analytical super resolution image approaches. Altogether, MSSR is a powerful, flexible, and generic tool for multidimensional and live cell imaging applications.

Key Words: super-resolution microscopy, diffraction limit, single frame, Mean Shift, fluorescence microscopy, live-cell imaging.

\section{Introduction}

Super-resolution Microscopy (SRM), which encompasses a collection of

49 methods that circumvent Abbe's optical resolution limit, has dramatically increased our capability to visualize the architecture of cells and tissues at the molecular level. There are several approaches to SRM which vary in terms of the final attainable spatial and temporal resolution, photon efficiency, as well as in their capacity to image live or fixed samples at depth $[1,2]$. One class of techniques exceed the diffraction limit by engineering the illumination or the point spread function (PSF), such as SIM and STED [3-5]. These techniques can be used for live imaging although they require specialized hardware and dedicated personnel for maintenance and operation. Single-molecule localization methods (e.g., STORM, PAINT, PALM) [6-9] that localize individual emitters with nanometer precision require temporal analysis of several hundred-tothousands of images and are prone to error due to fast molecular dynamics within live specimens.

Some SRM computational methods have few or no demands on hardware or sample preparation and provide resolution improvements beyond the diffraction limit [10-13]. The quantity and performance of computational methods have both increased over the past decade given the many advantages they present, such as their low barriers to entry and generic applicability to data acquired with any microscopy modality (wide- 
66 field, confocal, or light-sheet). However, these methods also present some limitations,

67

68

69 such as the possible introduction of artifacts [14], the requirement for high signal-tonoise ratio (SNR) data and the acquisition of tens to hundreds of frames [10-13], which limit their applicability to reconstruct fast dynamical processes.

Here, we introduce the $\underline{\text { Mean }} \underline{\text { Shift }} \underline{\text { Super-Resolution principle for digital images }}$ 'MSSR' (pronounced as messer), derived from the Mean Shift (MS) theory [15, 16]. MSSR improves the resolution of any single fluorescence image up to 1.6 times, including its use as a resolution enhancement complement after the application of other super-resolution methods. Additionally, we demonstrate the super-resolving capabilities of MSSR as a standalone method for a variety of fluorescence microscopy applications, through a single-frame and temporal stack analysis, allowing resolution improvements towards a limit of $40 \mathrm{~nm}$.

Open-source implementations of MSSR are provided for ImageJ (as a plugin), $\mathrm{R}$, and MATLAB, some of which take advantage of the parallel computing capabilities of regular desktop computers (Supplementary Note 7). The method operates almost free of parameters; users only need to provide an estimate of the point spread function (PSF, in pixels) of the optical system, choose the MSSR order, and decide whether a temporal analysis will take place (Supplementary material MSSR Manual). The provided opensource implementations of MSSR represent a novel user-friendly alternative for the bioimaging community for unveiling life at its nanoscopic level.

\section{Results}

The MSSR principle.

MSSR is tailored around the assumption that fluorescence images are formed by signals collected from point sources (i.e., fluorophores) convolved with the PSF of the microscope (Supplementary Notes 1, 2 and 3). Processing a single image with MSSR starts with the calculation of the MS, which guarantees that large intensity values on the diffraction-limited (DL) image coincide with large positive values in the MSSR image (Supplementary Note 4). Further algebraic transformations then restore the raw intensity distribution and remove possible artifacts caused by the previous step (edge effects and noise dependent artifacts), giving rise to an image that contains objects with a narrower full width at half maximum (FWHM) (Figure 1a). This procedure is denoted by $\operatorname{MSSR}^{0}$, 8 as the first stage to shrink emitter distribution. 
The MS is locally computed by a kernel window that slides throughout the entire image, subtracts the sample mean (weighted local mean) as well as the central value of the kernel using a spatial-range neighborhood (Supplementary Notes 2 and 3, Figure S4 and S5, Table S1) $[15,16]$. The MS is a vector that always points towards the direction of the intensity gradient and its length provides a local measure of the fluorescence density and brightness [17-19]. Since the MS lies within the gradient space, its values depend on the difference between the central pixel of the neighborhood and the surrounding pixels and thus is not necessarily linked to the fluorescence intensity values of the raw image. A mathematical proof, provided in Supplementary Note 4, demonstrates that the minimum MS value, computed from a Gaussian distribution, matches with the point of maximum intensity of the initial distribution (Supplementary Note 4, Figure S6).

The increase in resolution offered by $\mathrm{MSSR}^{0}$ was evaluated by the Rayleigh and Sparrow limits [20-22], which are two criteria that establish resolution bounds for two near-point sources (Figure 1b). Processing with $\mathrm{MSSR}^{0}$ of two-point sources located at their resolution limit (2.5 $\sigma$ and $2 \sigma$ for Rayleigh and Sparrow limit respectively, Figure $1 \mathrm{c}$ vertical discontinuous lines) decreases the dip (height at the saddle point) [23] within their intensity distributions (Figure $1 \mathrm{~b}$ and $1 \mathrm{c}$ ). Processing a single image with $\mathrm{MSSR}^{0}$ shifts the resolution limit by $26 \%$ and $20 \%$, according to the Rayleigh and Sparrow limits respectively (Figure 1c vertical continuous lines). Therefore, processing a single fluorescence image with $\mathrm{MSSR}^{0}$ will reduce the FWHM of individual emitters. Also, a comparison of the shrinkability of $\mathrm{MSSR}^{0}$ applied to Gaussian and Bessel distributions are shown in Figure S7. Additionally, the reduction of FWHM of Bessel distribution at different wavelengths of the visible spectrum are shown in Figure S8.

Since the result of MSSR is an image, we used the resulting image to seed an

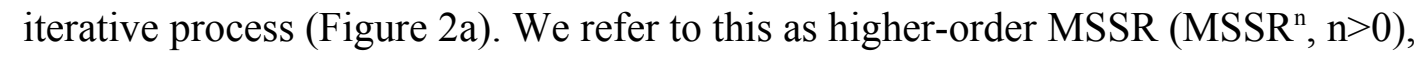
which delivers a further gain of resolution per $n$-iteration step (Figures. 2a and S9). As the order of $\mathrm{MSSR}^{\mathrm{n}}$ increases, both the FWHM of emitters (Figure S10) and the dip of their intensity distribution decrease (Figure 2b). Numerical approximations indicate that two point-sources separated at $1.6 \sigma$ are resolvable with $\mathrm{MSSR}^{3}$, but not when their separation is $1.5 \sigma$ (Figure $2 b$ ). The separation of $1.6 \sigma$ sets the theoretical resolution limit of $\mathrm{MSSR}^{\mathrm{n}}$. 
To empirically test the ability of $\mathrm{MSSR}^{\mathrm{n}}$ to achieve super-resolution image within a single frame, a commercial nanoruler sample (GATTA-SIM140B, GATTAquant) was imaged by Structured Illumination Microscopy (SIM) and widefield fluorescence microscopy, which was then processed by MSSR ${ }^{\mathrm{n}}$. The iterative processing of the widefield data with $\mathrm{MSSR}^{3}$ reveals the two fluorescence emitters located at a separation of $140 \mathrm{~nm}$ which is consistent with the result obtained by SIM (Figure 2c).

\section{MSSR further increases the resolution of super-resolved images.}

Based on the MSSR capabilities to generate a super-resolved micrograph after processing a single fluorescence image, we explored if a pre-existing super-resolved image can be further enhanced by MSSR.

First, we used temporal stack of DL images of tubulin-labeled microtubules collected at high fluorophore density [24] (previously used to test and compare a variety of SRM algorithms) [25], which were subject to ESI, SRRF or MUSICAL analysis [1113], where each was used to compute a single super-resolved image (Figure 3a). Supplementary note 9 contains an in-depth comparison of MSSR reconstructions combined with other SRM analytical methods (ESI, SRRF and MUSICAL), which achieve super-resolution through a temporal analysis [12-14]. Post-processing of ESI, SRRF or MUSICAL images with $\mathrm{MSSR}^{0}$ increases contrast and resolution (Figure 3a).

Second, a sequence of images of randomly blinking emitters placed along a synthetic tubular structure [26] was processed with $\operatorname{MSSR}^{0}$ after analysis with MUSICAL. In both reconstructions, three regions (small squares in Figure 3b) were chosen to assess the gain in resolution, visualized in terms of the distance between the normalized intensity distributions peaks. MSSR further resolves the edges of the synthetic structures on the MUSICAL-processed image without changing the position of the distribution peaks (Figure $3 b$ ) as predicted by our theory.

Lastly, we applied MSSR on a super-resolved SIM image of sister chromatids of mouse chromosomes (Figure 3c). Similar to the results obtained above, processing with MSSR increases both the contrast and resolution of the final image. Each of the individual SRM methods tested performs optimally under specific experimental 
162

163

164

165

166

167

168

169

conditions; one can thus choose whichever of them to use based on the available infrastructure, optical setup, and biological or experimental conditions that best fit the specific research goals. Altogether these data show that post-processing with MSSR increases resolution by a factor up to 1.6 times of any tested super-resolution technique.

\section{Temporal analysis of MSSR}

In theory, MSSR can be applied to a sequence of images (Supplementary Note 5). Based on the increase in resolution offered by computational methods that rely on temporal analyses (SRRF, ESI, MUSICAL), we investigated whether a further resolution gain could be achieved by applying a temporal analysis to a sequence of single frame MSSR images (t-MSSR ${ }^{\mathrm{n}}$ ) (Figure 4a). Pixel-wise temporal functions $(P T F)$, such as average (Mean), variance (Var), the temporal product mean (TPM), coefficient of variation $(\mathrm{CV})$ or auto-cumulant function of orders 2 to $4\left(\mathrm{SOFI}_{2}, \mathrm{SOFI}_{3}\right.$, $\mathrm{SOFI}_{4}$ ) [10], can be used to create an image with enhanced spatial resolution (Supplementary Note 5, Table S2).

To experimentally validate the increase in resolution from single-frame (sf$\mathrm{MSSR}^{\mathrm{n}}$ ) to t-MSSR${ }^{\mathrm{n}}$, we used two different nanoruler systems, an in-lab CRISPR/ $\mathrm{dCas} 12 \mathrm{a}$ nanoruler, used to score nanoscopic distances between individual fluorescent sites down to $100 \mathrm{~nm}$, and a commercial nanoruler with fluorophores positioned at 40 $\mathrm{nm}$ of separation (GATTA-PAINT, 40G, and 40RY. Gattaquant).

The CRISPR/dCas12a nanoruler system consists of a dsDNA with four binding sites for dCas12a uniformly distributed every $297 \mathrm{bp}$ (equivalent to $\sim 100 \mathrm{~nm}$ of separation) (Figure S33a). To validate this system, we imaged the association of the CRISPR/dCas 12 a complex to the binding sites on the dsDNA by atomic force microscopy (AFM) and measured the distance between each dCas12a complex (Figure S33b).

The CRISPR-dCas12a nanorulers were then imaged in buffer by total internal reflection fluorescence microscopy (TIRFM) for further MSSR analysis. We used a DNA-PAINT approach for fluorescence indirect tagging [27], in which a fluorescent ssDNA probe hybridizes with an extension of the gRNA. The "blinking" of the fluorescence signal is attained by events of association and dissociation between the fluorescent probe and the gRNA on the CRISPR/dCas12a nanoruler at the binding site. 
In the DL image, amorphous spot-like fluorescent patterns were observed (Figure 4b). sf-MSSR ${ }^{3}$ processing of either an isolated frame or an average projection of the corresponding stack of 100 images (DL-AVG) could not resolve individual CRISPR/dCas $12 \mathrm{a}$ binding sites (Figure $4 \mathrm{~b}$ ), and only after processing by t-MSSR ${ }^{3}$ did individual binding sites became resolved (Figure 4c). The result of t-MSSR ${ }^{3}$ varied in relation to the temporal function used (Figure 4c). The best result for this nanoruler was obtained by the pixel-wise temporal variance ( $V a r)$ of the $s f-M_{S S R}^{3}$ stack (Figure $4 c$ ). t$\mathrm{MSSR}^{3}$-Var resolved nearby emitters engineered to recognize binding sites located at $100 \mathrm{~nm}$ (Supplementary Movie S1), provided by scoring association-dissociation events between the imaging probe and the gRNA.

To determine the distance between two dCas12a sites along the DNA chain we obtained the distribution of distances between dCas12a binding sites taking in consideration their unidimensional association to a semi-flexible polymer such as the DNA [28]. Estimated distances after t-MSSR ${ }^{3}$-Var in the CRISPR/dCas12a nanoruler are $85 \pm 14 \mathrm{~nm}, 152 \pm 21 \mathrm{~nm}, 232 \pm 37 \mathrm{~nm}$ (Figure 4d). These results confirm that t$\mathrm{MSSR}^{3}$ can successfully resolve nanoscopic distances.

To explore the resolution limit attainable by $\mathrm{t}-\mathrm{MSSR}^{\mathrm{n}}$ even further, we looked at a nanoruler system with smaller separation between fluorophore sites (from Gattaquant) (Figure S34a). Analysis with t-MSSR ${ }^{3}$ of 100 images revealed individual fluorescent spots at $40 \mathrm{~nm}$ apart (Figure 4e and Supplementary Figure S34b). The data presented in Figure $4 \mathrm{e}$ demonstrate that $\mathrm{t}-\mathrm{MSSR}^{3}$ resolves nanoscopic distances in the $30-80 \mathrm{~nm}$ range, validating a lower experimental spatial resolution bound of $0.5 \sigma(\approx 40 \mathrm{~nm})$, which depends on the emission wavelength of the fluorophore (Figure 4e,

Supplementary Figure S8c). In comparison, SRRF, ESI and MUSICAL were not able to resolve fluorescent emitters located $40 \mathrm{~nm}$ apart, consistent with their limit within the range of 50-70 $\mathrm{nm}$ (Figure 4f) [11-13].

\section{Single frame nanoscopy, free of noise-dependent artifacts}

The theory of image processing by MSSR (Supplementary Note 5), suggests that it should be robust over a wide range of SNR, granted by four factors. First, when processing a single frame, MS works as a local spatial frequency filter (a smoothing filter); regions corresponding to the image background are homogenized by the kernel window, reducing variation in background noise. Second, one of the steps of the MSSR 
226 procedure is to remove the MS negative constraints. This threshold operation exerts

227 influence on structures at $\sigma$, at about $65 \%$ of the intensity distribution of the emitters;

228 values below this threshold will be considered as noise and set to zero value. Third,

229 when using a PTF, nanoscopic information is enriched due to temporal oversampling of

230 the hidden fluorescent structure. Fourth, the spatial kernel of the MSSR algorithm

231 operates within the subpixel realm; the number of neighboring pixels is digitally

232 increased through bicubic interpolation providing digital oversampling of the emitters'

233 locations (Supplementary Note 6).

We then experimentally assessed the capacity of MSSR to denoise fluorescence images and determine whether it introduces noise-related artifacts. We used a PSFcheck slide [29], which contains an array of regular fluorescent nanoscopic patterns shaped by laser lithography (Figure 5). Analysis with $\mathrm{sf}^{-\mathrm{MSSR}^{\mathrm{n}}}$ or t-MSSR ${ }^{\mathrm{n}}$ showed, in comparison to alternative approaches, striking denoising capabilities without introducing noticeable artifacts (Figure 5a) (Supplementary Note 9). These artifacts, resembling amorphous nanoscopic structures around the fluorescent ring or within it, were commonly found at reconstructions generated by other analytical techniques (Figure S22).

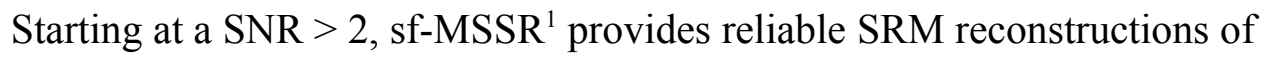
comparable quality to other SRM approaches, which demand the temporal analysis of the fluorescence dynamics (Figure 5a and Supplementary Note 9). We quantified the quality of the reconstructions by calculating the Resolution Scaled Pearson (RSP) coefficient and the Resolution Scaled Error (RSE), which provide a global measurement of the quality of the reconstruction by comparing the super-resolution image and the reference image (in this case, the DL image) [14]. Higher RSP and lower SRE values are associated with reliable reconstructions (Supplementary Note 8). When the SNR is above 5, all tested algorithms perform similarly well in quality (Figure 5b), but their global errors differ from each other (Figure 5c). As expected, the RSE increased as a function of the SNR of the input images for any tested algorithm (Figure 5c).

Furthermore, the performance of MSSR in achieving a satisfactory reconstruction was assessed by varying the number of input images using a temporal analysis scheme (Supplementary Note 8). With SNR $>2$ input data, RSP reaches near maxima values and RSE near minima values when processing a single frame (Figure S19-20, Supplementary Movie S2). However, when computing MSSR using low SNR 
input data (SNR 2) a temporal analysis is required as RSP and RSE values reach a plateau only when a temporal stack of as few as 20 images is used (Figure S20-21, Supplementary Movie S3). These findings illustrate that the minimal number of frames needed by MSSR to provide a reliable reconstruction depends on the information itself, i.e., on the SNR and on the photophysical properties of the specimen (movies S1 - S3); and can be determined by computing RSP and RSE as function of the number of analyzed frames with t-MSSR ${ }^{\mathrm{n}}$ (Figure S21).

\section{Nanoscopic resolution with conventional fluorescence imaging}

To showcase the versatility of MSSR to super-resolve data acquired from different fluorescence applications, we evaluated its performance over a collection of experimental scenarios (Supplementary Note 10).

Analysis with MSSR provided nanoscopic resolution of rotavirus replication machineries (Figure S25), which were recently described by Garcés et al as a layered array of viral protein distributions [30]. Originally, it took the authors several days to weeks to generate a single super-resolution image by means of analyzing several stacks of hundreds of DL images using 3B-ODE SRM. With MSSR, we were able to achieve comparable results, through analyzing single DL frames within seconds with a regular desktop computer with either sf-MSSR ${ }^{1}$ or t-MSSR ${ }^{1}$ (Supplementary Note 7).

Mouse sperm cells are used to study the acrosomal exocytosis (AE), a unique secretory process which results from fusion events between the plasma membrane and a specialized vesicle called acrosome located in the sperm head [31,32]. Nanoscopic remodeling of both plasma membrane and actin cytoskeleton was imaged during the $\mathrm{AE}$ by means of sf-MSSR ${ }^{1}$, showing single frame temporal resolution (of milliseconds) (Figs. S26). At the onset of the AE, the FM4-64 fluorescence (a probe that fluoresces when bound to membranes) was confined to the plasma membrane and was visible above of a F-actin cytoskeleton fringe. During the AE, several fenestration events were observed to occur at both the plasma and acrosome membranes, as consequence of that, a notorious increase of FM4-64 was observed close-bellow the F-actin fringe (Supplementary Movie S5 a-f). The AE is a dynamic remodeling process that takes minutes to occur, sf-MSSR ${ }^{1}$ allows the observation of events occurring at the millisecond scales, which are hindered when using other SRM multi-frame analytical approaches, such as SRRF or 3B [11,33], due to their mandatory need of a temporal 
analysis of the fluorescence dynamics to unveil nanoscopic detail (compare Figures S26 and S27).

Background noise is known to be an important issue in single-particle tracking (SPT) applications as it decreases the ability to faithfully localize particles and follow them through time $[34,35]$. Moreover, the spatial overlap of PSFs derived from individual particles makes it challenging for SPT algorithms to recognize them as separate entities. The denoising capabilities of $\mathrm{sf}_{-} \mathrm{MSSR}^{1}$ enhanced both the contrast and spatial resolution of freely diffusing in-silico particles previously used as benchmarks to test a variety of SPT algorithms (Figure S28) [36]. Pre-processing of the images with sfMSSR $^{1}$ improved the tracking performance of three commonly employed SPT tracking algorithms: (i) the LAP framework for Brownian motion as in [37, 38], (ii) a linear motion tracker based on Kalman filter [39-41], and (iii) a tracker based on Nearest neighbors [42-44] within a wide range of particle densities and SNR (Figure S29). Additional testing with $\mathrm{sf}-\mathrm{MSSR}^{\mathrm{n}}$ showed an increase in nanoscopic colocalization accuracy in double imaging experiments in single-molecule DNA curtain assays (Figure S30) [45].

Plasmalemma- and nuclear-labeled transgenic Arabidopsis thaliana plants are routinely used to study cell fate and proliferation during root development in time-lapse confocal microscopy experiments in two and three dimensions [46, 47]. When applied to lateral root primordium cells, located deep inside the parent root, sf-MSSR ${ }^{1}$ demonstrated the capacity to achieve multidimensional nanoscopic resolution as it revealed isolated nanodomains resembling nucleosome clutches, previously reported in mammalian cells $[48,49]$, within the nuclei of a lateral root primordium cells (Figure S31 and Supplementary Movie S10). Similar observations were performed upon epidermal root tissues visualized via selective plane illumination microscopy (SPIM) after examination of volumetric data with sf-MSSR $^{1}$ (Figure S32). In combination, these studies provide evidence for the capabilities of MSSR to resolve biological detail at nanoscopic scales using either simple or advanced fluorescence microscopy technologies. 


\section{Discussion}

- Novel theoretical contributions

From the historical point of view, since the seminal development of the MS theory $[15,16]$ and until the present day, few statistical and imaging applications based on the theory of MS compute the MS vector itself [50]. This can be explained, in part, because previous applications of MS are based on finding modes in the features space and did not operate directly in the derivative space. In contrast, MSSR represents an application of MS theory that operates in the second derivative space. By computing the MS vector and estimating densities among pixels, MSSR computes a probability function for the fluorophore estimates whose individual fluorescence distributions are narrowed in comparison with the PSF of the optical system. The exploration of the information stored on the second derivative space of the image can be also achieved by substituting the MS by similar functions that operate in such space, e.g., Laplacian, Hessian, Difference of Gaussians [51] which, in comparison with the MS, offer computational advantages as they can be expressed in the Fourier space and implemented using the FFT algorithm [51]. The information harbored in the second derivative space of the DL image is used by MSSR to compute a super-resolved image with higher spatial frequencies than the corresponding DL image, hence, overcoming both the Rayleigh and Sparrow limits, and setting up an undescribed limit of resolution which deserves further exploration and characterization.

The MS theory is not restricted by the number of dimensions of the information required to compute the kernel windows over which MSSR operates (Supplementary notes 2 and 3). Given that, MSSR parameters are suitable to extend its application to assess data with higher dimensions. For example, in 2D images, the spatial parameter of MSSR, which encompasses the lateral resolution width of the PSF, is defined to be the same in the $\mathrm{x}$ and $\mathrm{y}$ dimensions of the image. In such case, the shape of the kernel is circle- or square-like, depending on the application used. For three-dimensional (3D) microscopy imaging, the lateral ( $\mathrm{x}-\mathrm{y}$ plane) and axial ( $\mathrm{x}-\mathrm{z}$ and $\mathrm{y}-\mathrm{z}$ planes) dimensions are affected in different ways by diffraction. The MSSR principle can be further extended for explicit volumetric imaging by means of using an asymmetric kernel which can be defined following the 3D lateral-axial aspect ratio of the PSF. In addition, the definition of the spatial kernel can be refined to also consider possible deformations of axial symmetry of the PSF due to optical aberrations introduced by the imaging 
357

358

359

system or by the sample itself. A similar reasoning aimed to extend the portfolio of applications of MSSR can be envisaged considering spatial-range parameter, the latter narrowing down the working intensity space where local calculations of MSSR take place.

\section{Novel contributions to microscopy}

We present a new SRM approach capable of achieving multidimensional nanoscopy through single-frame analysis under low SNR conditions and with minimal noisedependent artifacts. Limited only by the imaging speed of the optical system setup, MSSR increases resolution by analyzing either a single frame, or by applying MSSR to each individual image in a stack followed by the application of a pixel-wise temporal function. MSSR is a powerful stand-alone method for either single or multi-frame SRM approaches, or as post-processing method which can be applied to other analytical multi-frame (restricted to camera-based systems) or hardware dependent SRM methods for further enhancement of resolution and contrast. We demonstrated MSSR compatibility with other SRM methods and showed that its usage improved resolution and overall image quality in all the cases tested.

SRM analytical multi-frame approaches such as SRRF, ESI, MUSICAL and 3B demand a temporal analysis which limits their utility for multi-dimensional imaging of live samples [46]. The need to collect hundreds to thousands of images of the same pseudo-static scene, challenges the applicability of these methods in multidimensional imaging. The temporal multi-frame requirement imposes a tradeoff between the achievable temporal and spatial resolutions. MSSR removes these constraints while maintaining computational efficiency (Supplementary note 7).

\section{We present applications of the MSSR principle that revealed fast molecular} dynamics through single-frame analysis of live-cell imaging data, with reduced processing times in comparison with similar SRM approaches (Supplementary notes 7 and 10). Moreover, MSSR greatly improves the tracking efficacy of SPT methods by means of reducing background noise and increasing both the contrast and SNR of noisy SPT movies, enhancing the ability to resolve the position of single emitters. MSSR further pushes the limits of live-cell nanoscopy by its excellent single-frame performance. This flexibility extends its utility to most fluorescence microscopy and alternative SRM methods. 
Achieving both high (or sufficient) temporal and spatial resolution within a

390

391 broad range of fluorescence microscopy applications is a common goal among the bioimaging community. With recent advances in microscopy equipment and imaging protocols, the gap between the highest attainable resolution in the temporal and spatial dimensions within the same experiment, has narrowed. This has been a challenge especially because both parameters often involve mutually exclusive optical instrumentation and experimental strategies. The introduction of MSSR represents one more step in the right direction as it drastically reduces the amount of data needed to reconstruct a single super-resolved micrography.

No longer having to sacrifice either temporal or spatial resolution over the other, has led some scientists to propose new ways to analyze imaging data. Some approximations have been tailored to study millisecond molecular dynamics and structural feature changes within the same experiment [52], e.g., by taking advantage of the simultaneous use of image correlation spectroscopy (ICS) and SRM methods such as SRRF [11]. In these contexts, MSSR could improve the analysis in three ways: a) it delivers reliable SRM images in low SNR scenarios, which are common in the experimental regimes of ICS due to the relatively fast frame rates of its applications, $b$ ) MSSR introduces no noise-dependent artifacts which further refines the quality of the spatial analysis and c) since no temporal binning is necessary for MSSR, there is no restriction in the level of temporal detail retrievable from the ICS analysis.

Sub-millisecond time-lapse microscopy imaging can now be achieved by sCMOS technologies, with applications for particle velocimetry [53], rheometry [54], and optical patch clamp [55]. We envisage further applications for MSSR in these areas through unveiling nanoscopic detail hidden in single DL images. Moreover, MSSR can facilitate correlative nanoscopic imaging through crosstalk with other imaging techniques such as electron microscopy, i.e., CLEM: correlative light electron microscopy [56]; or atomic force microscopy, i.e., CLAFEM: Correlative light atomic force electron microscopy [57]. In addition, MSSR can be applied to nanoscopic volumetric imaging by using it together with expansion microscopy [58], oblique angle microscopy [59], SPIM, and lattice light sheet microscopy [60], extending their capabilities to previous unattainable resolution regimes.

A recent study by Chen $\mathrm{R}$. et al., suggests that deep-learning based artificial intelligence $(\mathrm{AI})$ can reconstruct a super-resolution image from a single frame of a DL image [61]. Such AI-based SRM approaches are promising, however, they are limited to 
423 the existence of a maximum likelihood image obtained with another SRM, such as

424 STORM, that is required for neural network training an error minimization. Otherwise,

425 the method it is prompted to bias the final reconstruction toward the topological

426 information used to train the AI - network [61]. Our approach works completely

427 independent of other SRM methods and provides evidence of the existence of a new

428 resolution limit which lies on the second derivative space of the DL image, information

429 inaccessible when using neural networks.

430 MSSR applications might impact far beyond the field of microscopy, as its

431 principles can be applied to any lens-based system such as astronomy [62] and high-

432 resolution satellite imagery [63]. 
434

435

436

437

438

439

440

441

442

443

444

445

446

447

448

449

450

451

452

453

454

455

456

457

458

459

460

461

462

463

464

465

466

467

468

469

\section{References}

1 Galbraith, C. G. \& Galbraith, J. A. Super-resolution microscopy at a glance, .J. cell science, 124, 1607-1611, (2011).

2 Thorley, J. A., Pike, J. \& Rappoport, J. Z. Chapter 14 - Super-resolution Microscopy: A Comparison of Commercially Available Options. In Cornea, A. \& Conn, P. M. (eds.) Fluorescence Microscopy, 199-212, (Academic Press, Boston, 2014).

3 Hell, S. W. \& Wichmann, J. Breaking the diffraction resolution limit by stimulated emission: stimulated-emission-depletion fluorescence microscopy. Opt. Lett. 19, 780782, (1994).

4 Gustafsson, M. G. Surpassing the lateral resolution limit by a factor of two using structured illumination microscopy. J. microscopy 198, 82-87, (2000).

5 Wegel, E. et al. Imaging cellular structures in super-resolution with SIM, STED and Localisation Microscopy: A practical comparison. Sci. Reports 6, 27290, (2016).

6 Betzig, E. et al. Imaging intracellular fluorescent proteins at nanometer resolution. Science 313, 1642-1645, (2006).

7 Bock, H. et al. Two-color far-field fluorescence nanoscopy based on photoswitchable emitters. Appl. Phys. B 88, 161-165, (2007).

8 Oi, C., Mochrie, S. G. J., Horrocks, M. H. \& Regan, L. Paint using proteins: A new brush for super-resolution artists. Protein Sci. 29, 2142-2149, (2020).

9 Oi, C. et al. LIVE-PAINT allows super-resolution microscopy inside living cells using reversible peptide-protein interactions. Commun. Biol. 3, 458, (2020).

10 Dertinger, T., Colyer, R., Iyer, G., Weiss, S. \& Enderlein, J. Fast, background-free, 3d super-resolution optical fluctuationimaging (sofi). Proc. Natl. Acad. Sci. 106, 2228722292, (2009).

11 Gustafsson, N. et al. Fast live-cell conventional fluorophore nanoscopy with Image J through super-resolution radial fluctuations. Nat. Commun. 7, 12471, (2016).

12 Agarwal, K. \& Machán, R. Multiple signal classification algorithm for super-resolution fluorescence microscopy. Nat. Commun. 7, 13752, (2016).

13 Yahiatene, I., Hennig, S., Müller, M. \& Huser, T. Entropy-Based Super-Resolution Imaging (ESI): From Disorder to FineDetail.ACS Photonics 2, 1049-1056, (2015).

14 Culley, S.et al. Quantitative mapping and minimization of super-resolution optical imaging artifacts. Nat. Methods 15, 263-266, (2018).

15 Fukunaga, K. \& Hostetler, L. The estimation of the gradient of a density function, with applications in pattern recognition. IEEE Transactions on Inf. Theory 21, 32-40, (1975).

16 Yizong Cheng. Mean shift, mode seeking, and clustering.IEEE Transactions on Pattern Analysis Mach. Intell. 17, 790-799, (1995). 
17 Comaniciu, D. and Meer, P., Mean shift: a robust approach toward feature space analysis, IEEE Transactions on Pattern Analysis Mach. Intell. 24, 603-619 (2002).

18 Emami, E., Fathy, M. and Kozegar, E. Online failure detection and correction for camshifttracking algorithm, 2013 8th Iranian Conference on Machine Vision and Image Processing (MVIP), (2013), pp. 180-183.

19 Fazekas, F. J., Shaw, T. R., Kim, S., Bogucki, R. A. \& Veatch, S. L. A mean shift algorithm for drift correction in localization microscopy. Preprint at https://doi.org/10.1101/2021.05.07.443176 (2021).

20 Rayleigh, L. On the theory of optical images, with special reference to the microscope. J. Royal Microsc. Soc. 23, 474-482, (1903).

21 Sparrow, C. M. On Spectroscopic Resolving Power. Astrophys. J. 44, 76, (1916).

22 Diaspro, A. \& Bianchini, P. Optical nanoscopy. La Rivista del Nuovo Cimento 43, 385 $455,(2020)$.

23 Sharma, K.Optics: Principles and Applications (Academic Press, Elsevier Science, 2006).

24 Dataset: Tubulin 2d high density. http://bigwww.epfl.ch/smlm/challenge2013/.

25 Sage, D. et al. Super-resolution fight club: assessment of 2D and 3D single-molecule localization microscopy software. Nat. Methods 16, 387-395, (2019).

26 Dataset: Bundled tubes high density. http://bigwww.epfl.ch/smlm/challenge2013/.

27 Schnitzbauer, J., Strauss, M. T., Schlichthaerle, T., Schueder, F. \& Jungmann, R. Superresolution microscopy with DNA-PAINT. Nat. Protoc. 12, 1198-1228, (2017).

28 Wang, H. \& Milstein, J. N. Simulation Assisted Analysis of the Intrinsic Stiffness for Short DNA Molecules Imaged with Scanning Atomic Force Microscopy. PLOS ONE 10, 1-11, (2015).

29 Corbett, A. D.et al. Microscope calibration using laser written fluorescence. Opt. Express 26, 21887-21899, (2018).

30 Garcés Suárez, Y. et al. Nanoscale organization of rotavirus replication machineries. elife 8, e42906, (2019).

31 Romarowski, A. et al. Super-resolution imaging of live sperm reveals dynamic changes of the actin cytoskeleton during acrosomal exocytosis. J. Cell Sci. 131, (2018).

32 Balestrini, P. A. et al. Seeing is believing: Current methods to observe sperm acrosomal exocytosis in real time. Mol. Reproduction Dev. 87, 1188-1198, (2020).

33 Cox, S. et al. Bayesian localization microscopy reveals nanoscale podosome dynamics. Nat. Methods 9, 195-200, (2012).

34 Jin, S., Haggie, P. M. \& Verkman, A. S. Single particle tracking of membrane protein diffusion in a potential: Simulation, detection, and application to confined diffusion of $\mathrm{cftr}$ cl-channels. Biophys. J. 93, 1079-1088, (2007). 
506

507

508

509

510

511

512

513

514

515

516

517

518

519

520

521

522

523

524

525

526

527

528

529

530

531

532

533

534

535

536

537

538

539

540

541

35 Yamashita, N. et al. Three-dimensional tracking of plus-tips by lattice light-sheet microscopy permits the quantification of microtubule growth trajectories within the mitotic apparatus. J. Biomed. Opt. 20, 1 - 18, (2015)

36 Chenouard, N. et al. Objective comparison of particle tracking methods. Nat. Methods 11, 281-289, (2014).

37 Jaqaman, K., Loerke, D., Mettlen, M., Kuwata, H., Grinstein, S., Schmid, S. L. and Danuser, G. Robust single-particle tracking in live-cell time-lapse sequences, Nat. Methods 5, 695 (2008).

38 Fields, A. P. and Cohen, A. E. Optimal tracking of a brownian particle, Opt. Express 20, 22585-22601 (2012).

39 Kalman, R. E. A New Approach to Linear Filtering and Prediction Problems, J. Basic Eng.82, 35-45 (1960).

40 Yüce M. Y., Erdoğan, A., Jonáš, A. \& Kiraz, A. Single molecule tracking with kalman filtering. Frontiers in Optics 2011/Laser Science XXVII, FTuH5, (Optical Society of America, 2011).

41 Tinevez, J.-Y., Perry, N., Schindelin, J., Hoopes, G. M., Reynolds, G. D., Laplantine, E., Bednarek, S. Y., Shorte, S. L. and Eliceiri, K. W. Trackmate: An open and extensible platform for single-particle tracking, Methods 115, 80-90 (2017).

42 Patel, M., Leggett, S. E., Landauer, A. K., Wong, I. Y., and Franck, C. Rapid, topologybased particle tracking for high-resolution measurements of large complex 3D motion fields, Sci. Reports 8, 5581 (2018).

43 Gross, J., Köster, M. \& Krüger, A. Fast and efficient nearest neighbor search for particle simulations. Computer Graphics and Visual Computing CGVC, 55-63, (The Eurographics Association, 2019).

44 Cheezum, M. K., Walker, W. F., and Guilford, W. H. Quantitative Comparison of Algorithms for Tracking Single Fluorescent Particles, Biophys. J. 81, 2378-2388 (2001).

45 Calcines-Cruz, C., Finkelstein, I. J. \& Hernandez-Garcia, A. Crispr-guided programmable self-assembly of artificial virus-like nucleocapsids. Nano Lett. 21, 2752-2757, (2021).

46 Federici, F., Dupuy, L., Laplaze, L., Heisler, M. \& Haseloff, J. Integrated genetic and computation methods for in planta cytometry. Nat. Methods 9, 483-485, (2012).

47 Torres-Martínez, H. H., Hernández-Herrera, P., Corkidi, G. \& Dubrovsky, J. G. From one cell to many: Morphogenetic field of lateral root founder cells in arabidopsis thaliana is built by gradual recruitment. Proc. Natl. Acad. Sci. 117, 20943-20949, (2020).

48 Ricci, M. A., Manzo, C., García-Parajo, M. F., Lakadamyali, M., \& Cosma, M. P. (2015). Chromatin fibers are formed by heterogeneous groups of nucleosomes in vivo. Cell, 160, $1145-1158$. 
49 Rutowicz, K., Lirski, M., Mermaz, B., Teano, G., Schubert, J., Mestiri, I., Kroten, M., A., Fabrice,T. N., Fritz, S., Grob, S., Ringli, C., Cherkezyan, L., Barneche, F., Jerzmanowski, A., and Baroux, C. Linker histones are fine-scale chromatin architects modulating developmentaldecisions in arabidopsis, Genome Biol. 20, 157 (2019).

50 Wu, G., Zhao, X., Luo, S. \& Shi, H. Histological image segmentation using fast mean shift clustering method. BioMedical Eng. OnLine 14, 24, (2015).

51 Szeliski, R. Computer vision: Algorithms and applications (Springer Science \& Business Media, 2011).

52 Sankaran, J. et al. Simultaneous spatiotemporal super-resolution and multi-parametric fluorescence microscopy. Nat. Commun. 12, 1748, (2021).

53 Lin, Y.-H., Chang, W.-L., and Hsieh, C.-L. Shot-noise limited localization of single 20 $\mathrm{nm}$ gold particles with nanometer spatial precision within microseconds, Opt. Express 22, 9159-9170 (2014).

54 Glover, Z. J., Ersch, C., Andersen, U., Holmes, M. J., Povey, M. J., Brewer, J. R. and Simonsen, A. C. Super-resolution microscopy and empirically validated autocorrelation image analysis discriminates microstructures of dairy derived gels," Food Hydrocoll. 90, 62-71(2019).

55 Betzig, E. Nobel lecture: Single molecules, cells, and super-resolution optics, Rev. Mod. Phys. 87, 1153-1168 (2015).

56 Liss, V., Barlag, B., Nietschke, M. and Hensel, M. Self-labelling enzymes as universal tags for fluorescence microscopy, super-resolution microscopy and electron microscopy, Sci. Reports5, 17740 (2015).

57 de Boer, P., Hoogenboom, J. P. \& Giepmans, B. N. G. Correlated light and electron microscopy: ultrastructure lights up! Nat. Methods 12, 503-513, (2015).

58 Cahoon, C. K. et al. Superresolution expansion microscopy reveals the three-dimensional organization of the Drosophila synaptonemal complex. Proc. Natl. Acad. Sci. 114, 68576866, (2017).

59 Kim, J., Wojcik, M., Wang, Y., Moon, S., Zin, E.A., Marnani, N., Newman, Z.L., Flannery, J.G., Xu, K. and Zhang, X. Oblique-plane single-molecule localization microscopy for tissues andsmall intact animals, Nat. Methods 16, 853-857 (2019).

60 Hu, Y. S., Zimmerley, M., Li, Y., Watters, R. and Cang, H. Single-molecule superresolution light-sheet microscopy," ChemPhysChem 15, 577-586 (2014).

61 Chen, R., Tang, X., Shen, Z., Shen, Y., Li, T., Wang, J., Cui, B., Guo, Y., Du, S. and Yao, S. Deep-learning super-resolution microscopy reveals nanometer-scale intracellular dynamics at the millisecond temporal resolution, Preprint at https://doi.org/10.1101/2021.10.08.463746 (2021). 
62 Puschmann, K. G. \& Kneer, F. On super-resolution in astronomical imaging. Astronomy \& Astrophysics 436, 373-378, (2005).

58063 Guo, R., Shi, X., Zhu, Y. \& Yu, T. Super-resolution reconstruction of astronomical images using time-scale adaptive normalized convolution. Chin. J. Aeronaut 31, 17521763, (2018). 


\section{Figures}

584

a MSSR order 0

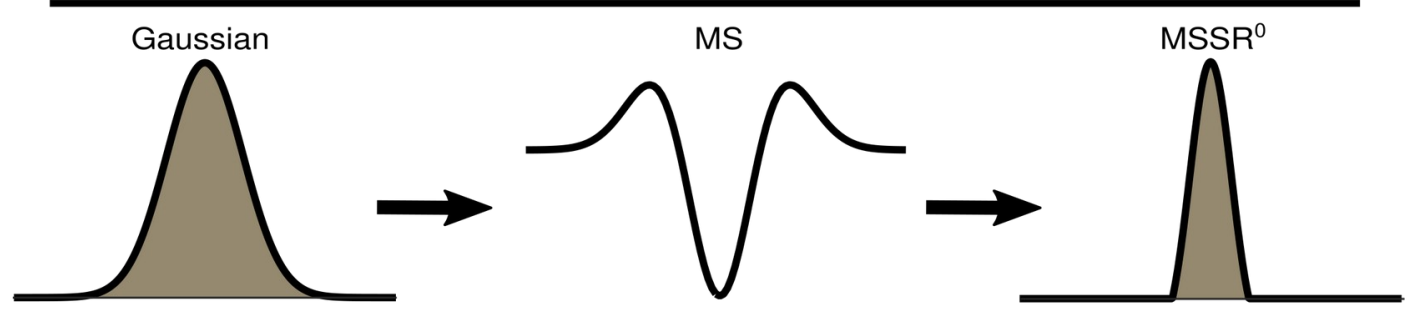

b Sparrow limit

Rayleigh limit
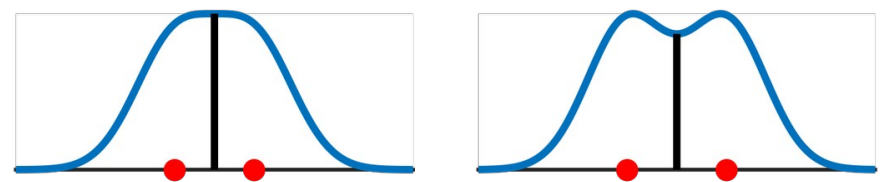

Sum of Gaussians
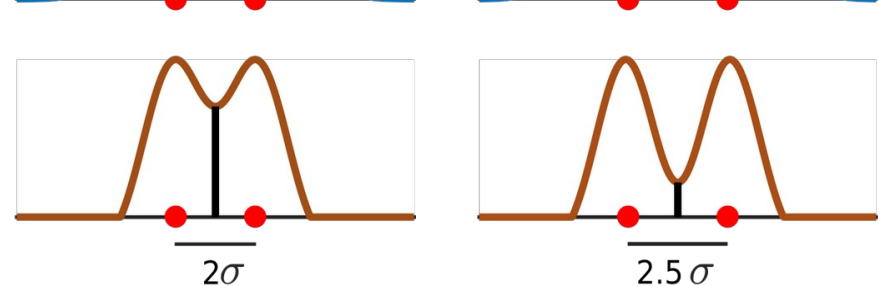

$-\mathrm{MSSR}^{0}$

Dip

- Emitter position

c

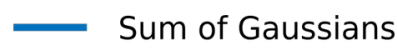

- MSRR $^{0}$

$\operatorname{MSRR}^{0}$ Rayleigh limit

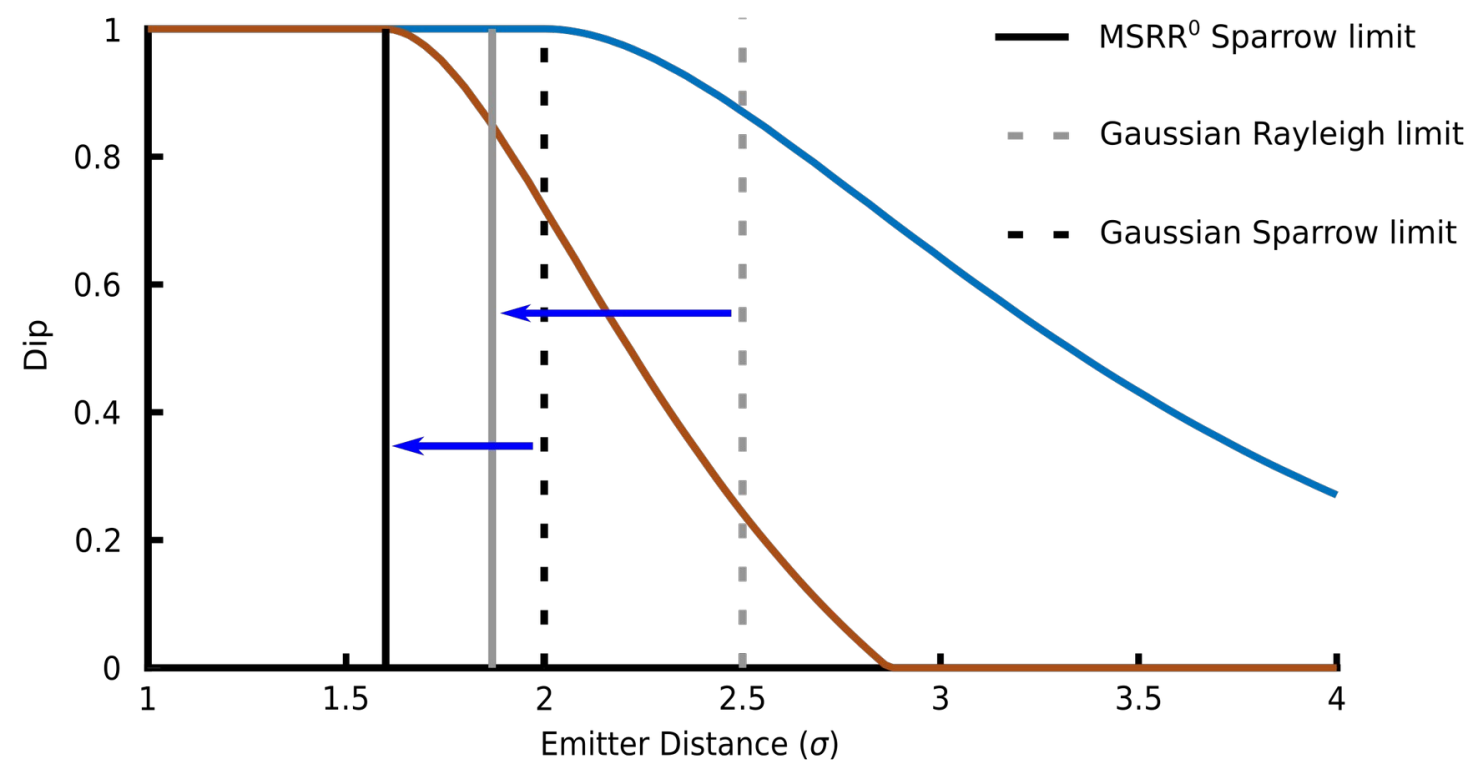

586 Figure 1. MSSR of zero order increases resolution by reducing the width of the spatial distribution of photons emitted from modelled fluorescent emitters. a) The

588 MS is applied to the initial Gaussian distribution of photons emitted by a point-source

589 (left) resulting in a MS graph (center). Application of further algebraic transformations 
590 (see Supplementary Note 5 and Figure S7 (ii-iv)) provides the $\mathrm{MSSR}^{0}$ distribution

591 (right). b) Sparrow and Rayleigh limits (blue, diffraction-limited) and the corresponding

$592 \mathrm{MSSR}^{0}$ transformation (brown) for two point-sources. Red dots represent each emitter's

593 location. The dip is indicated by a vertical black line. The inter-emitter distance is

594 expressed as $\sigma$-times their individual standard deviation before MSSR processing. c)

595 Dip computed for two point-source emitters of Gaussian distribution located away at

596 distinct $\sigma$ (blue line) where the corresponding $\operatorname{MSSR}^{0}$ result is also depicted (red line).

597 For Gaussian: Rayleigh limit - gray discontinuous line, Sparrow limit - black

598 discontinuous line. For MSSR ${ }^{0}$ : Rayleigh limit - gray solid line, Sparrow limit - gray

599 solid line. The solid vertical lines represent the distance between emitters such that

600 when processed with $\mathrm{MSSR}^{0}$, the criterions of Rayleigh and Sparrow are obtained. 
bioRxiv preprint doi: https://doi.org/10.1101/2021 10.17.464398; this version posted October 24, 2021. The copyright holder for this
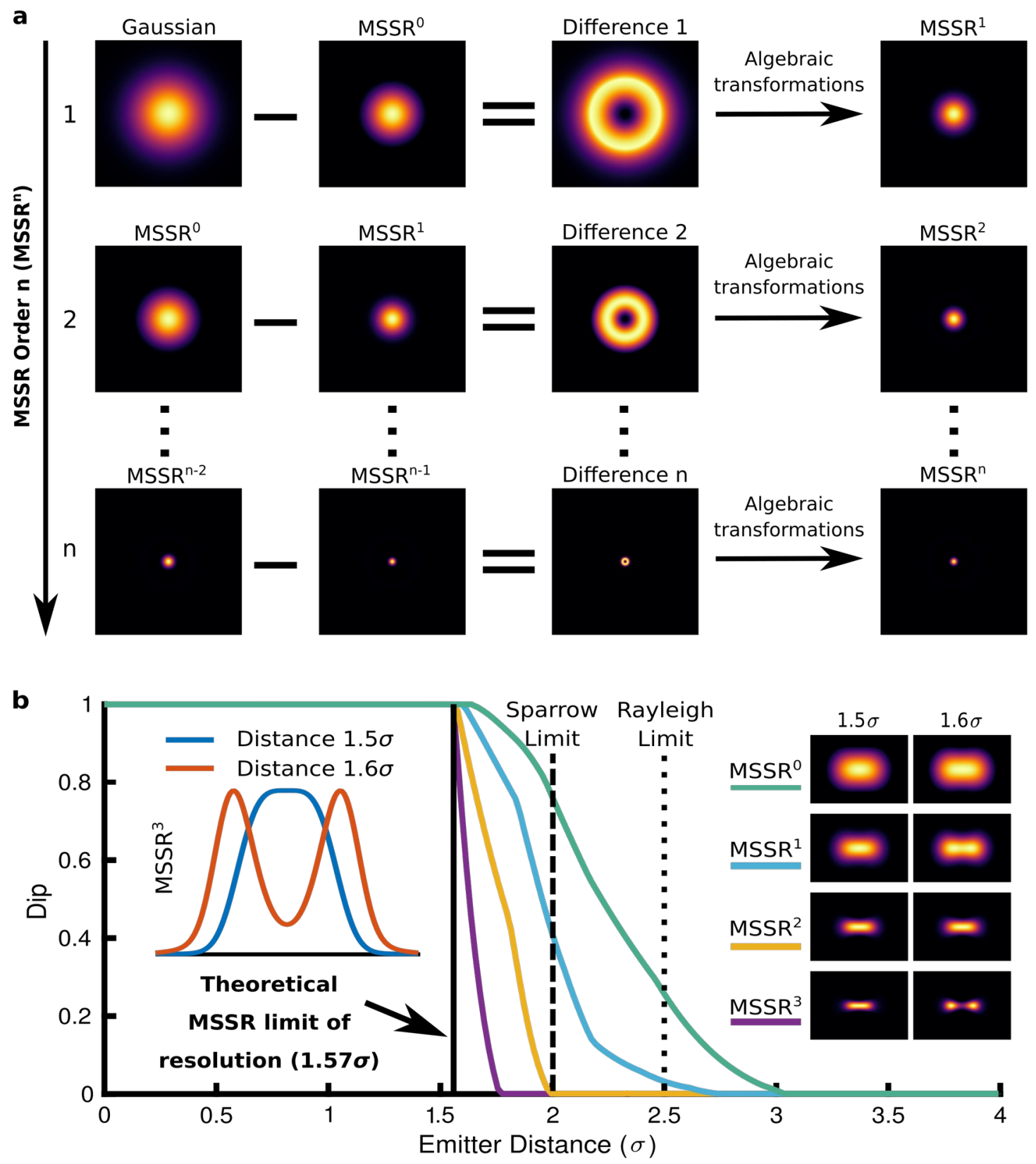

C GATTA-SIM $140 \mathrm{~nm}$ nanoruler (Diffraction-limited $+\mathrm{MSSR}^{\mathrm{n}}$ )
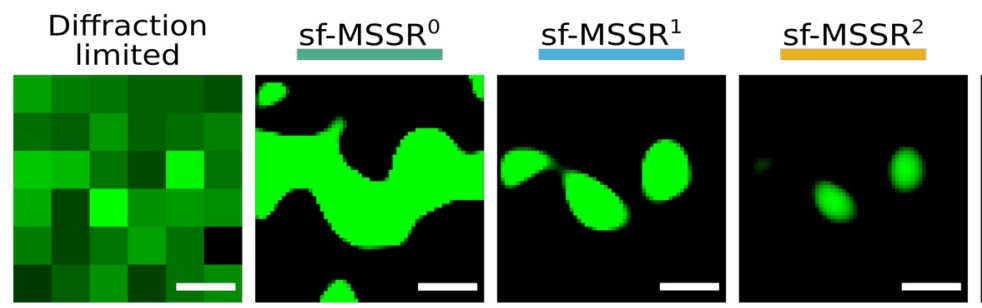

Sf-MSSR ${ }^{3}$

SIM
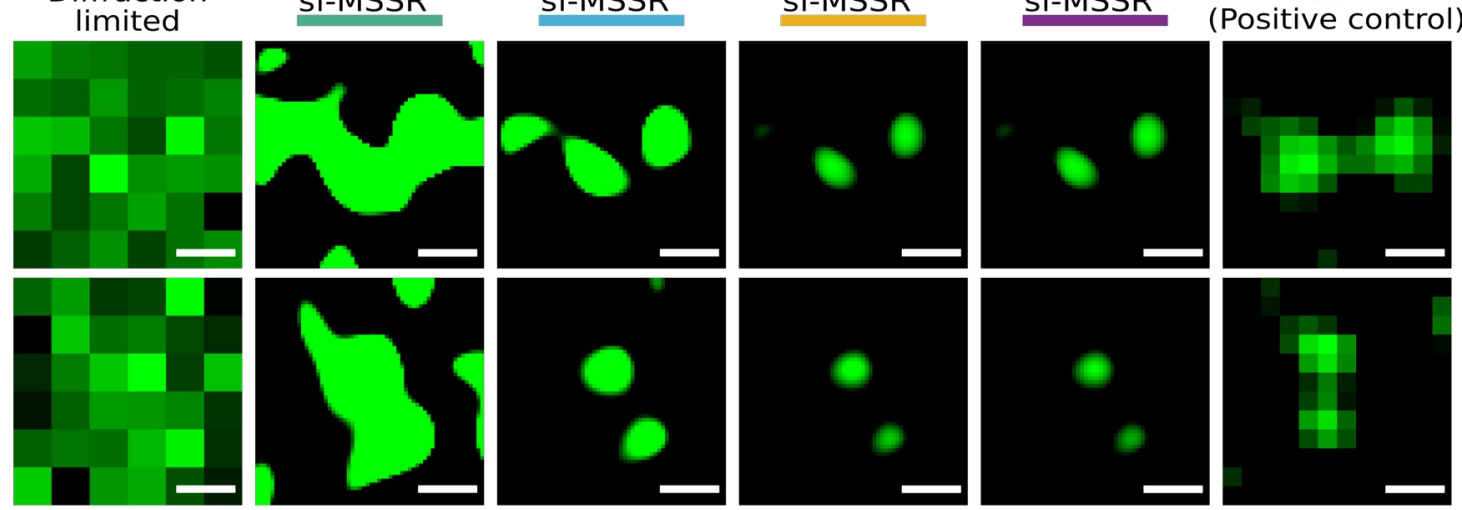
603 Figure 2. Single-frame MSSR analysis of higher order attains a resolution limit of

$6041.6 \sigma$ for nearby emitters. a) The algorithm for computing higher-order MSSR

$605\left(\mathrm{MSSR}^{\mathrm{n}}\right)$ is presented. The first iteration of MSSR $\left(\mathrm{MSSR}^{1}\right)$ is given by subtracting the

$606 \mathrm{MSSR}^{0}$ from the original image, resulting in a donut-like region centered at the

607 emitter's location. MSSR ${ }^{1}$ is computed after applying further algebraic transformations

608 (see Supplementary Note 5 and Figure S8 (ii-iv) for a full description of the $\mathrm{MSSR}^{\mathrm{n}}$

609 process). The second iteration encompasses the subtraction of $\mathrm{MSSR}^{1}$ from $\mathrm{MSSR}^{0}$ and

610 the same algebraic transformations as used for generation of MSSR ${ }^{1}$. The process is

611 repeated by updating consecutive MSSR images which generates higher MSSR orders.

612 b) Theoretical limit of resolution achievable by $\mathrm{MSSR}^{\mathrm{n}}$. Dip computed for two Gaussian

613 emitters in accordance with the variation of the inter-emitter distance (expressed as $\sigma$ -

614 times their standard deviation before MSSR processing). Colored lines represent the dip

615 of MSSR order, from 0 to 3, computed at a given $\sigma$ distance between emitters. Images

616 on the right are the bidimensional representation of the $\mathrm{MSSR}^{\mathrm{n}}$ processing for two

617 single emitters separated at distances of $1.5 \sigma$ and $1.6 \sigma$. Note that, for $1.5 \sigma$, emitters are

618 unresolved up to the third order of MSSR. c) Experimental demonstration of the

619 resolution increases attainable with higher order MSSR using the GATTA-SIM 140B

620 nanoruler system. The intensity distribution of the emitter shrinks, both in $\sigma$ and

621 intensity, as the order of the MSSR increases (Figure S8). Nearby emitters (Alexa

622 Fluor ${ }^{\circledR}$ 488) located $140 \mathrm{~nm}$ apart are resolved using $\mathrm{MSSR}^{1}, \mathrm{MSSR}^{2}$ and $\mathrm{MSSR}^{3}$ (right

623 side). SIM images collected from the same sample (distinct fields) are shown as a

624 positive control. Scale bar: $100 \mathrm{~nm}$. 

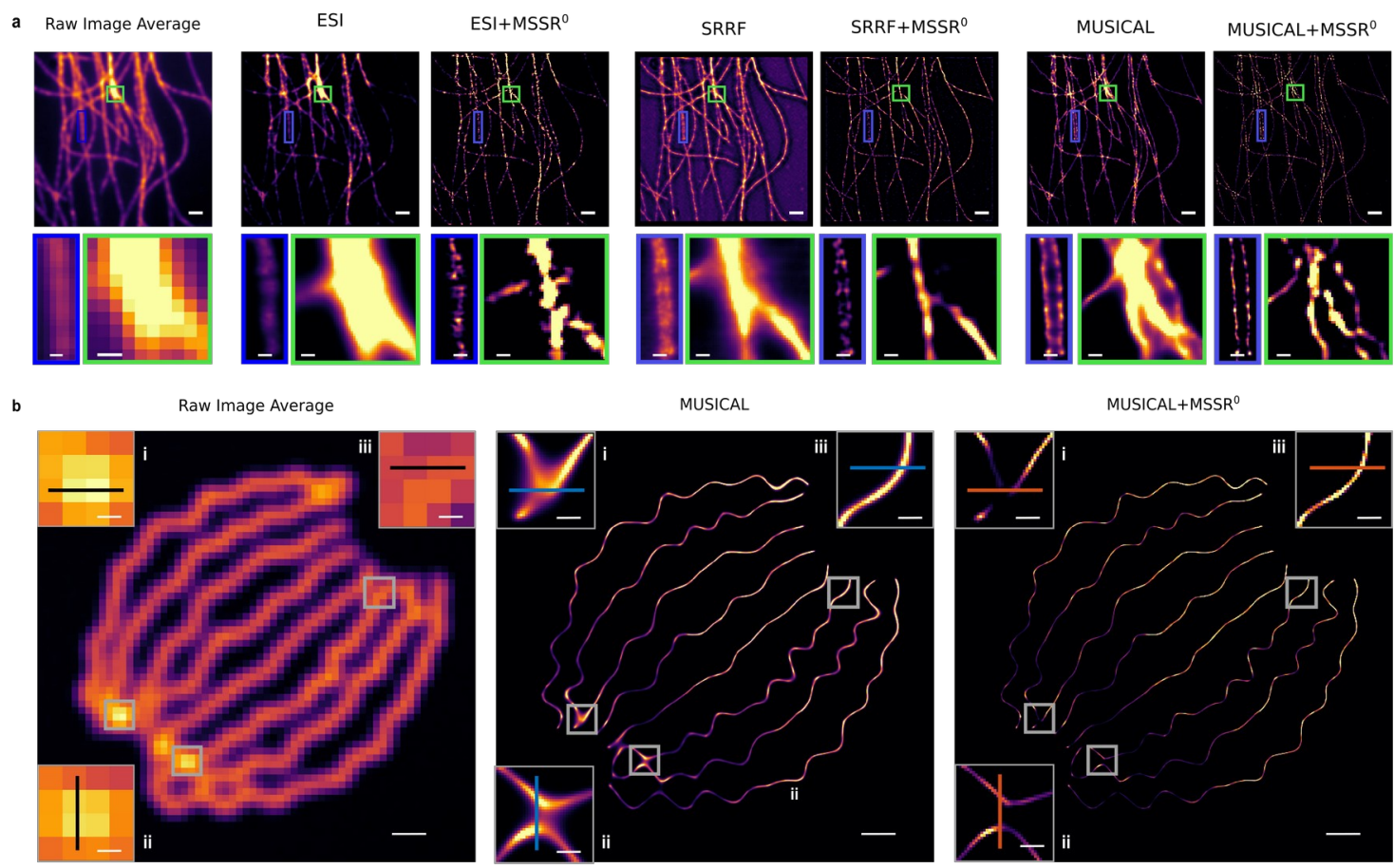

MUSICAL

MUSICAL+MSSR ${ }^{0}$
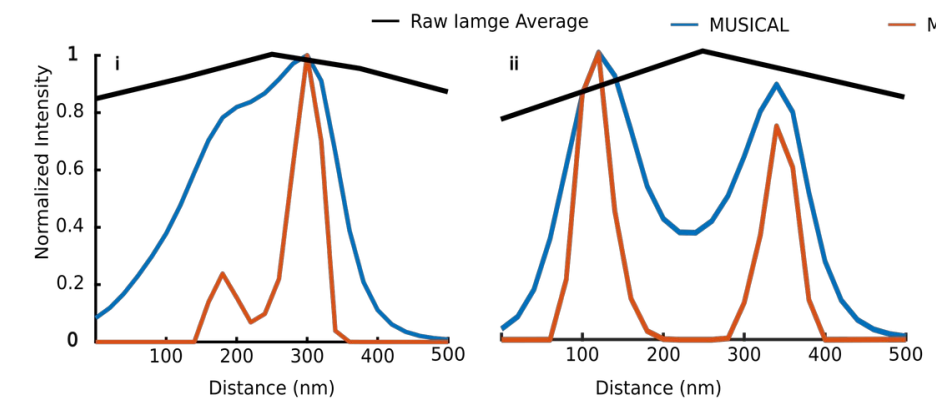

Diffraction Limited
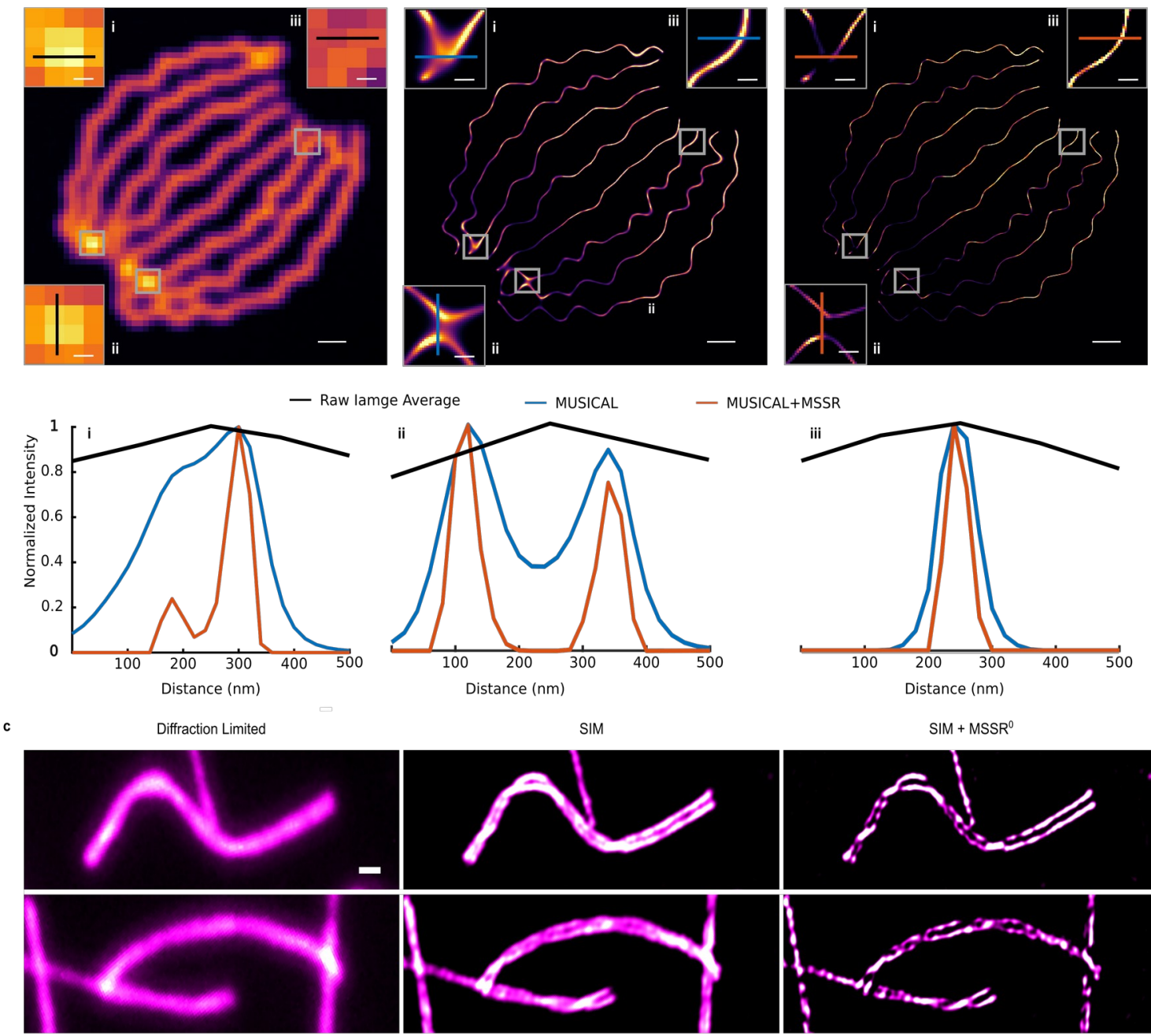

SIM
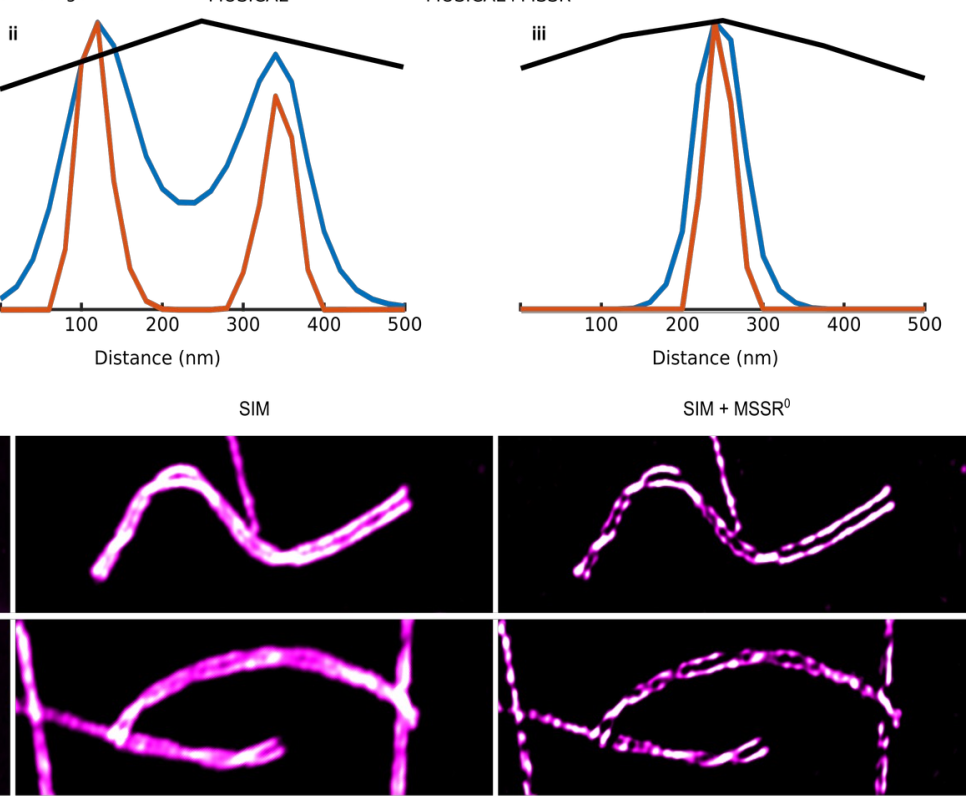

628 Figure 3. MSSR enhances resolution and contrast of single super-resolved images.

629 a) Comparison of SRM results of ESI, SRRF and MUSICAL alone and after post-

630 processing with $\operatorname{MSSR}^{0}\left(\mathrm{ESI}+\mathrm{MSSR}^{0}, \mathrm{SRRF}_{+} \mathrm{MSSR}^{0}, \mathrm{MUSICAL}+\mathrm{MSSR}^{0}\right)$, over a

631 temporal stack of 500 DL images of tubulin-labeled microtubules. The average

632 projection of the DL stack is shown on the leftmost side. b) Comparison of the increase

633 in spatial resolution of MUSICAL with and without post-processing with MSSR ${ }^{0}$

634 (MUSICAL $+\mathrm{MSSR}^{0}$ ), over a temporal stack of $361 \mathrm{DL}$ images of modelled

635 fluorophores bounded to a synthetic array of nanotubules (average projection shown on 
636 left). The graphs show the intensity profiles along the lines depicted in each of the insets

637 in the images of the upper row; black, blue and red lines correspond to the average DL,

638 MUSICAL and MUSICAL $+\operatorname{MSSR}^{0}$ images, respectively. c) Sister chromatids of

639 mitotic mouse chromosomes visualized by TIRFM (left), SIM (middle) and SIM +

$640 \operatorname{MSSR}^{0}$ (right). Scale bars: a) $1 \mu \mathrm{m}$, insets $=200 \mathrm{~nm}$; b) $500 \mathrm{~nm}$, insets $=100 \mathrm{~nm}$; c)

$641200 \mathrm{~nm}$.

642 

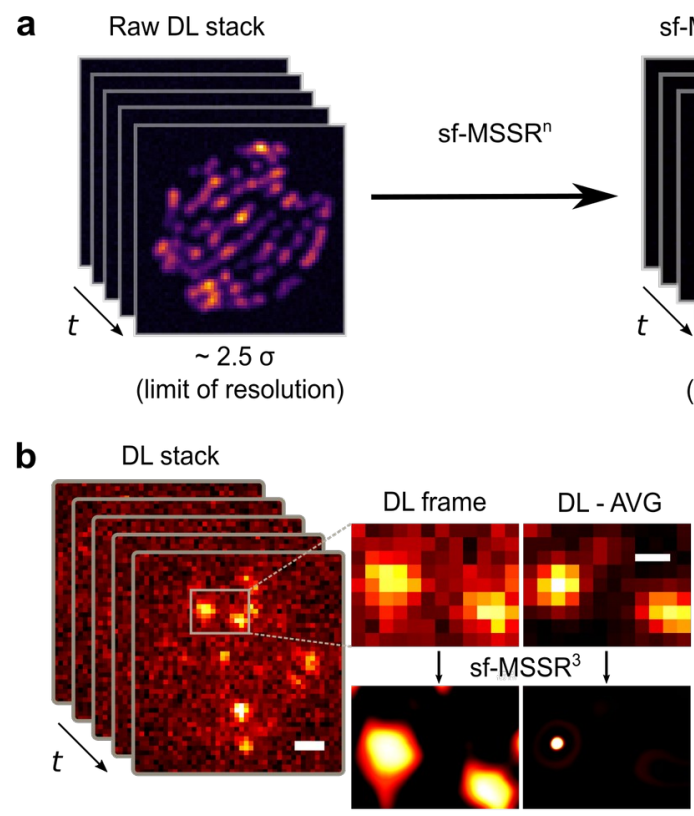

e

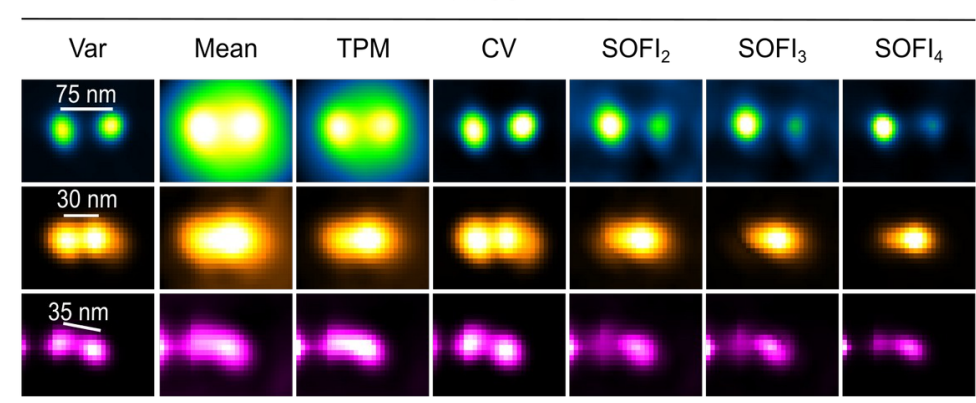

sf-MSSR ${ }^{\mathrm{n}}$ stack

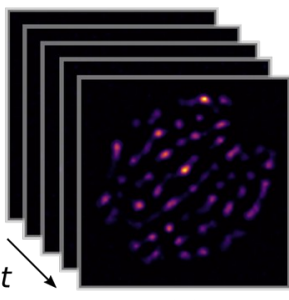

$\sim 1.6 \sigma$
(MSSR theoretical limit)

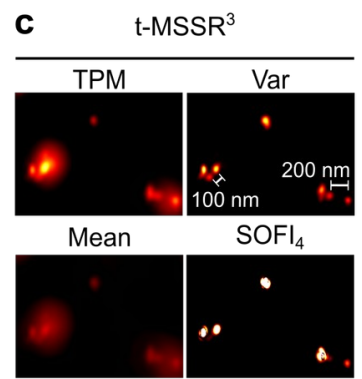

t-MSSR

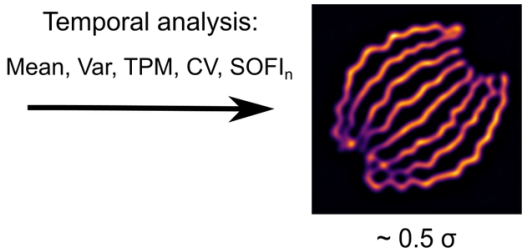

(further resolution gain)

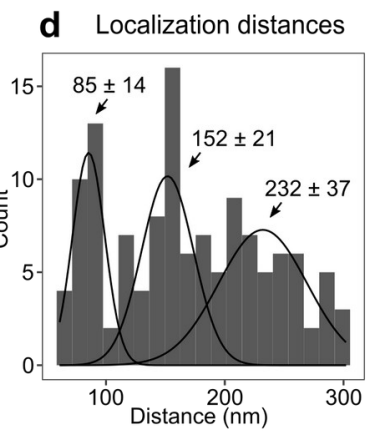

f

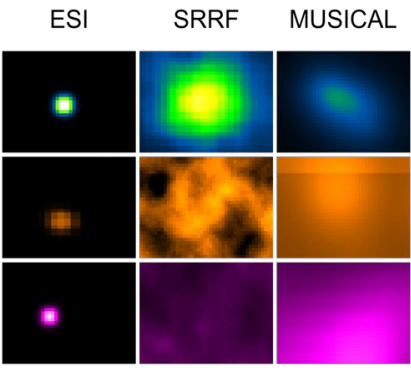

645

646

647

648

649

650

651

652

653

654

655

656

657
Figure 4. The temporal analysis of MSSR provides a further increase in resolution to approximately $40 \mathrm{~nm}$. a) Single-frame analysis of MSSR of a given order $n$ is applied to each frame of a sequence, becoming the sf-MSSR ${ }^{\mathrm{n}}$ stack. Next, a pixel-wise temporal function (PTF) converts the MSSR stack into a single super-resolved t-MSSR ${ }^{\mathrm{n}}$ image. Depending on the temporal entropy of the dataset and on the PTF used, a resolution enhancement can be obtained. b) Left: a stack of DL images of a CRISPR/dCas12a nanoruler system. Scale bar: $1 \mu \mathrm{m}$. Right: zoomed region of the first frame in the stack, along with the average projection (DL-AVG) of a stack of 100 images, before and after MSSR processing. Scale bar: $400 \mathrm{~nm}$. c) PTF applied to a stack of $\mathrm{MSSR}^{3}$ images (t-MSSR${ }^{3}$ ). Fluorescent emitters are separated by $100 \mathrm{~nm}$, as established by the CRISPR/dCas12a nanoruler system. Four types of PTF were computed: TPM, Var, Mean and $\mathrm{SOFI}_{4}$. d) Euclidean distances between nearby emitters automatically computed from t-MSSR ${ }^{3}$-Var images, following a worm-like chain model 
658 (16 regions of interest used, $1.5 \mu \mathrm{m}^{2}$ each). e) Comparison of the results obtained with

659 each of the PTF analysis available with MSSR (see Table S3), for a commercially

660 available GATTA-PAINT nanoruler system. The Var column shows inter-emitter

661 distances resolved in the range $30-75 \mathrm{~nm}$. Atto 488 (green), Atto 550 (orange) and Atto

662655 (magenta) fluorescent probes were used. f) Same nanorulers shown in e) but

663 analyzed with either ESI, SRRF or MUSICAL. 
a

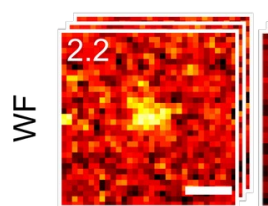

SNR low SNR high
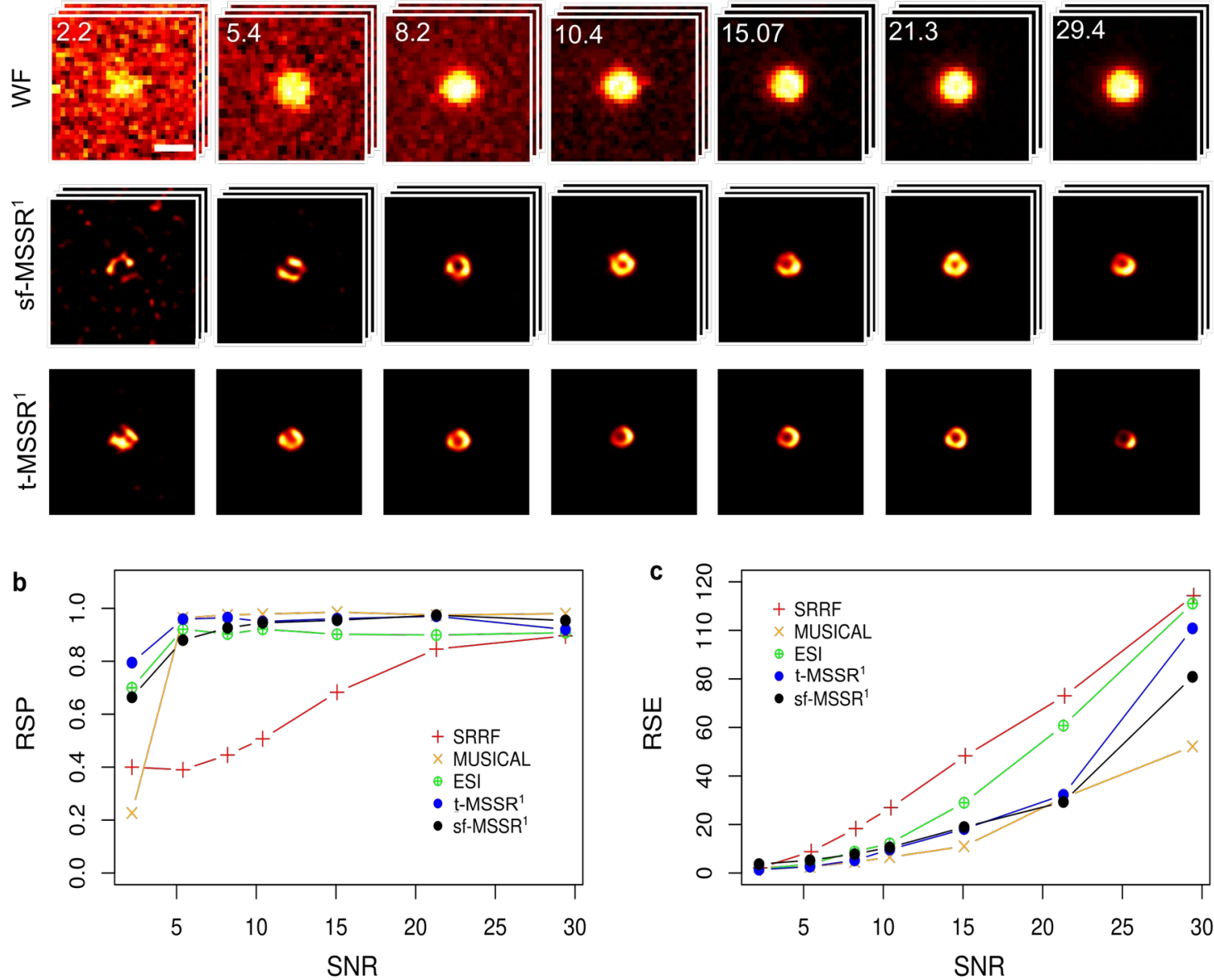

665 Figure 5. MSSR is robust to image noise and shows high global performance when

666 compared to other SRM analytical procedures. a) sf-MSSR ${ }^{1}$ and t-MSSR ${ }^{1}$ of 100

667 images provide consistent reconstructions across a wide range of SNR. The expected

668 feature is a uniform fluorescent ring located at the center of the image with a dark

669 background lacking fluorescence. Each image is displayed to show its full intensity

670 range. The row for DL images (Widefield, WF) exemplifies a stack of 100 frames

671 collected at the corresponding SNR. The central row represents a resolved stack using

672 sf-MSSR ${ }^{1}$. The third row shows the super-resolved micrography after t-MSSR ${ }^{1}$ analysis

673 of 100 DL images using TPM for temporal analysis (see table S2). Scale bar: $1 \mu \mathrm{m}$. b-c)

674 Resolution Scaled Pearson (RSP) coefficient (b) and Resolution Scaled Error (RSE) (c),

675 computed for the super-resolution reconstructions provided by SRRF, MUSICAL, ESI,

676 sf-MSSR ${ }^{1}$ and t-MSSR ${ }^{1}$ (100 frames). b) RSP measures a global correlation between

677 reconstruction and reference (input DL image), values closer to 1 indicate a reliable

678 reconstruction. c) RSE measures the absolute difference of the reconstructed image and 
679 its reference. Lower values of RSE at a particular SNR mean reduced global error in the 680 reconstruction. Scale bar: $1 \mu \mathrm{m}$. 


\section{Data availability}

683 All raw imaging data which support the findings of this study are available from the

684 corresponding author upon request. Source data are provided with this paper.

685 Correspondence and requests for materials should be addressed to A.G.

686

687 Code availability

688 Source code for R and MATLAB platforms is available as supplementary materials, the

689 MSSR plugin for FIJI/ImageJ is available at https://github.com/MSSRSupport/MSSR.

\section{Funding}

692 This research was supported by Dirección General de Asuntos del Personal Académico

693 (DGAPA) - Programa de Apoyo a Proyectos de Investigación e Innovación

694 Tecnológica-UNAM (PAPIIT). and by Mexican Consejo Nacional de Ciencia y

695 Tecnología (CONACyT) grant A1-S-9236 to JGD, grant number IN211821 to AG,

696 IN211216 to CDW, IN204221 to JGD. Microscopy equipment was provided and

697 maintained through CONACYT grants 123007, 232708, 260541, 280487, 293624 and

698 294781. MB thank to IBYME-CONICET: Grants: PICT 2017-3047 (Agencia Nacional

699 de Promoción de la Investigación, el Desarrollo Tecnológico y la Innovación),

700 Fundación Williams; Fundación Rene Barón. DK acknowledges the support of the

701 National Science Foundation grant 2102832.

702 We also acknowledge the Programa de Becas de Posgrado of CONACYT for 703 granting scholarships to ETG, RPC, AL, DM, VA, EBA, CCC, GVG, DT, HTM, JLM, $704 \mathrm{HH}$, and to JPO. YG acknowledges DGAPA/UNAM for postdoctoral fellowship.

\section{Acknowledgements}

707 To the Microscopy Facility at EMBL Rome and IBYME-CONICET for providing

708 samples and materials for experiments. To the ECE and ICIMAF institutes for

709 theoretical and experimental input to this work. The authors thank the LNMA staff for 
710 providing support with microscopy imaging. The OpenSPIM project was technically

711 executed and extended by Oliver Valdez Escalona. ET and AG thank Angelica Flores

712 Navarrete and Yuriney Abonza Amaro for their valuable feedback during the

713 elaboration of the manuscript.

715 Author contributions

716 Conceptualization: ET, AG.

717 Methodology: ET, AG, RP, AHC, RR., JGD.

718 Investigation: ET, AG, RP, AHC, DM, VA, EBA, CC, GV, AHG, DT, JM, YG, MaB, 719 MJ, HTM, HH, JO.

720 Visualization: ET, AG, HT.

721 Supervision: AG, AHC, DK.

722 Funding Acquisition: AG, AD, MB, JGD, CW.

723 Project Administration: AG.

724 Drafting Main Document: ET, AG, AL, AHC, CW.

725 Drafting Supplementary Material: ET, AG, AL, RR, JR, DK.

726 All authors contributed to the writing and reviewing of the manuscript.

728 Competing interests

729 The authors declare no competing interests.

730

731 Additional information

732 The online version contains supplementary methods, supplementary notes,

733 supplementary movies, the MSSR manual for FIJI/ImageJ, MSSR scripts R and

734 MATLAB, and additional references. 

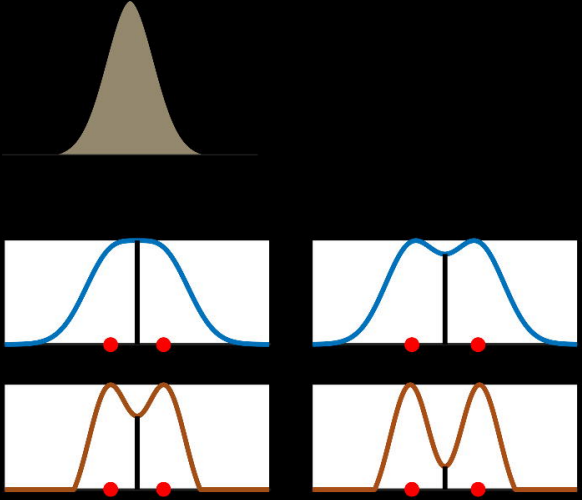

$\theta$
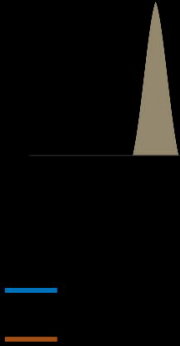

0
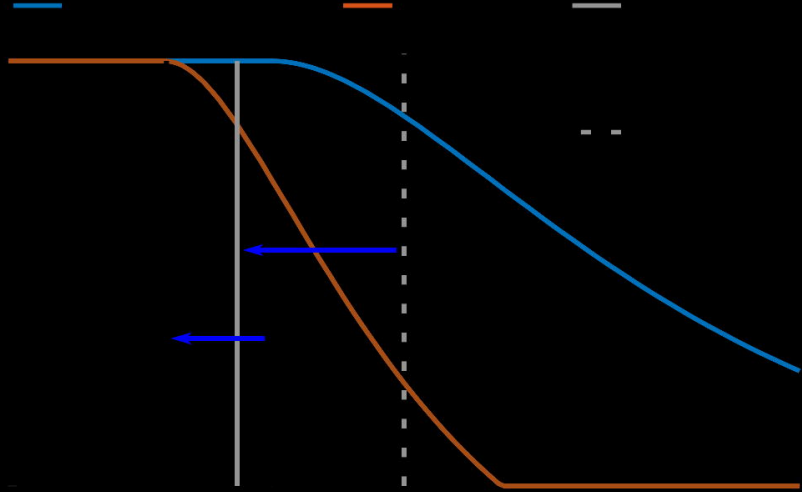

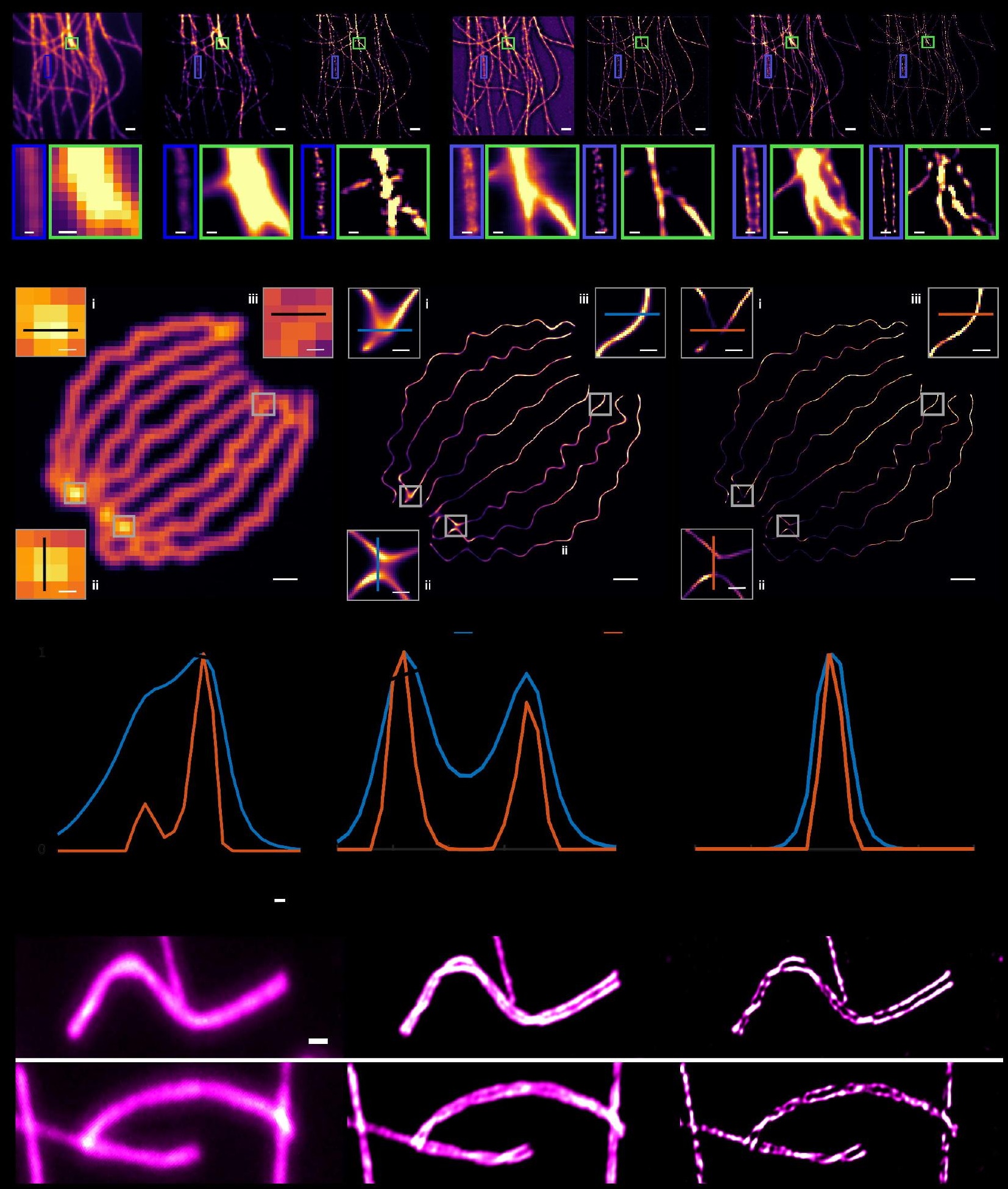

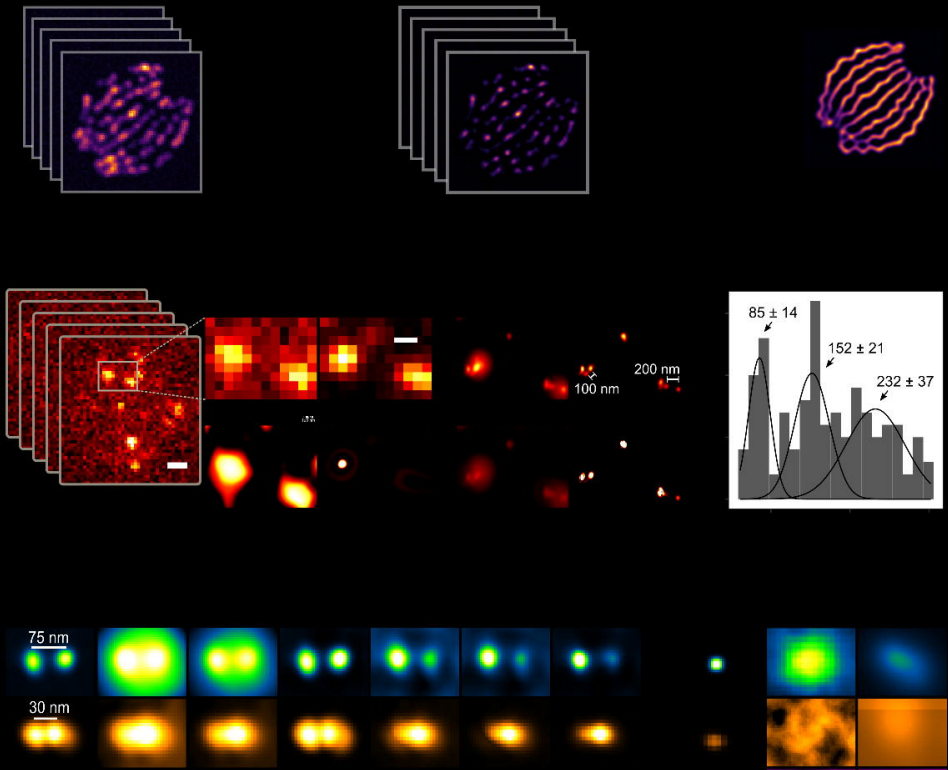

$35 \mathrm{~nm}$

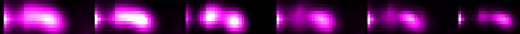



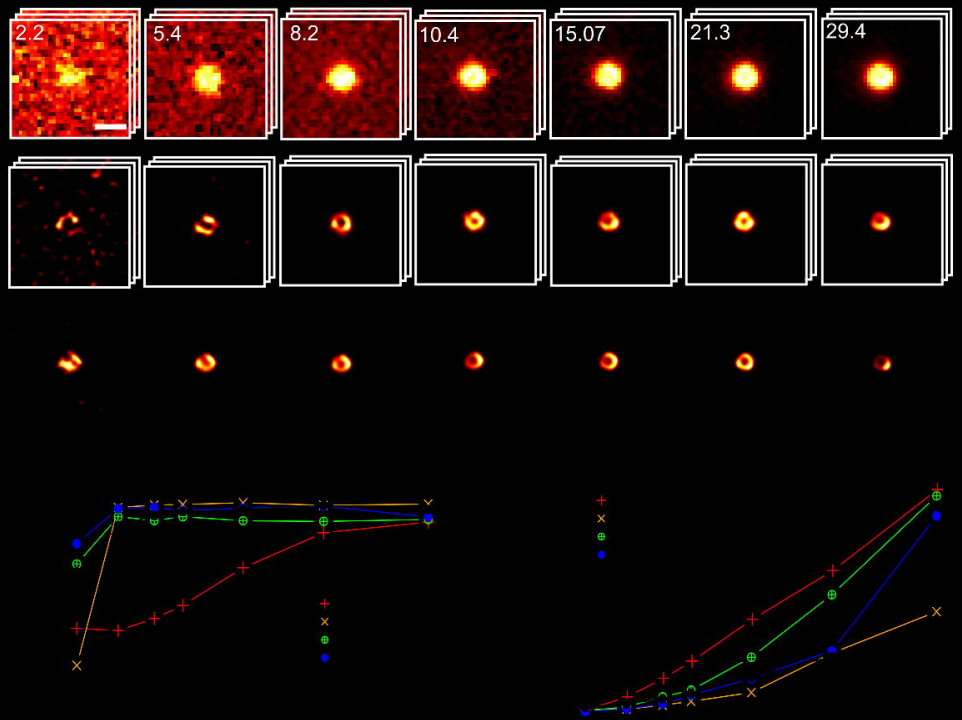